\title{
Applying Commercial Robotic Technology to Radioactive Material Processing
}

\author{
E. L. Grasz \\ R. Sievers
}

This paper was prepared for submittal to the IEEE International Conference on Robotics and Automation, Sacramento, CA, 4/7-12/91

\section{November 1990}

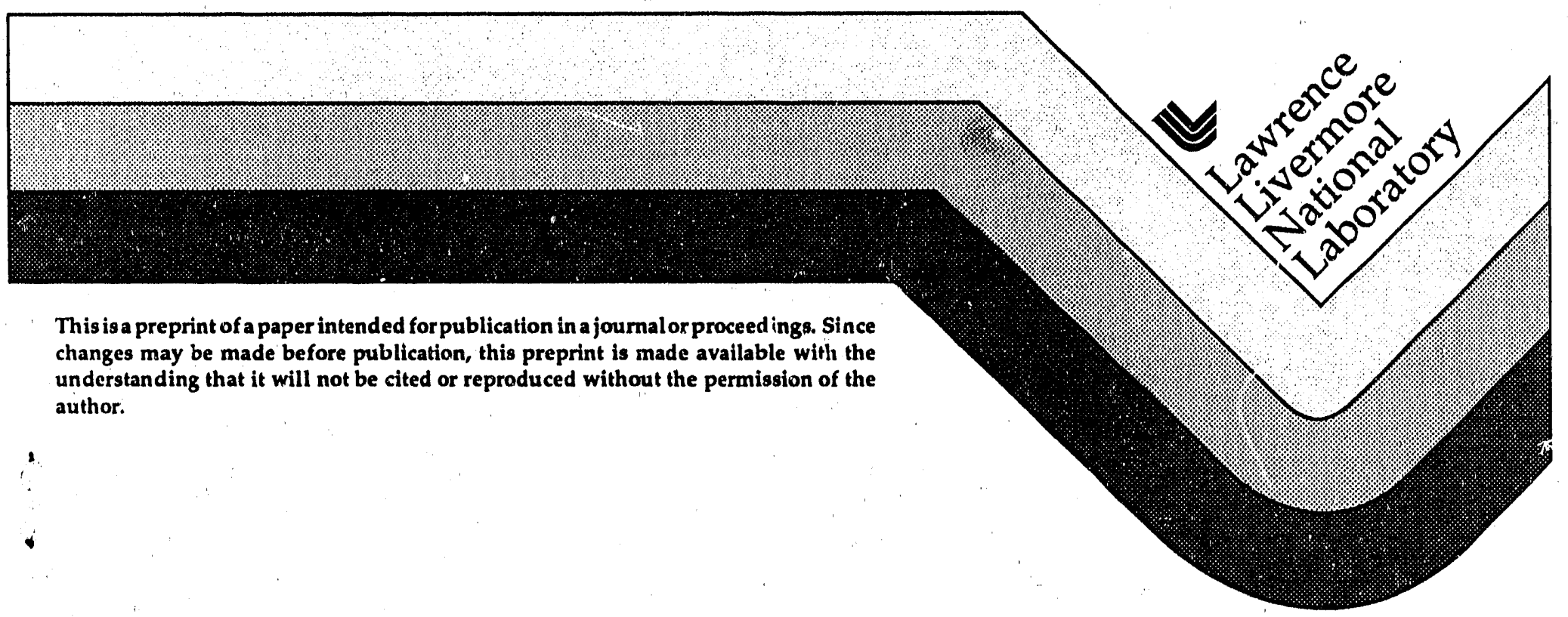




\section{DISCLAIMER}

This document was prepared as an account of work sponsored by an agency of the United States Government. Neither the United States Government nor the University of California nor any of their employees, makes any warranty, express or implied, or assumes any leyal liability or responsibility for the accuracy, completeness, or usefulness of any information, apparatus, product, or process disclosed, or represents that its use would not infringe privately owned rights. Reference herein to any specific

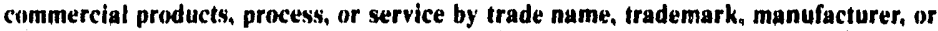
otherwise, does not necessarily constitute or imply its endorsement, recommendation, or favoring by the United States Government or the University of California. The views and opinions of authors expressed herein do not necessarily state or reflect those of the United States Government or the University of California, and shall not be used for advertising or product endorsement purposes. 


\title{
Applying Commercial Robotic Technology to Radioactive Material Processing
}

\author{
Erna L. Grasz, Lawrence Livermore National Laboratory \\ Ralph H. Sievers, Jr., P.E., Science Applications International Corporation
}

\section{Abstract}

The development of robotic systems for glove box process automation is motivated by the need to reduce operator radiation dosage, minimize the generation of process waste, and to improve the security of nuclear materials.

Commercial robotic systems are available with the required capabilities but are not compatible with a glove box environment. Alpha radiation, concentrated dust, a dry atmosphere and restricted work space result in the need for unique adaptations to commercial robotics. Implementation of these adaptations to commercial robotics require performance trade-offs. A design and development effort has been initiated to evaluate the feasibility of using a commercial overhead gantry robot for glove box processing. This paper will present the initial results and observations for this development effort.

\section{Introduction}

The processing of radioactive materials has typically been performed with direct hands-on operation. Stricter regulation for reducing operator radiation dosage levels, process waste generation, and improving the security of nuclear materials has motivated the implementation of robotics technology for automating glove box processes. The most logical approach is to adapt available robotic technologies to the special requirements of process automation. This adaptation process creates the need for unique capabilities. These capabilities must include high reliability, easy maintainability and serviceability, while keeping a high degree of flexibility to complete various tasks.
Of the Department of Energy's (DOE) 1000 processing glove boxes in operation; only two limited operation glove boxes employ robotic systems. The ultimate goal is the modernization, of all production facilities within the DOE Weapons Complex.

\section{Considerations for Process Automation}

The constraints of the glove box workspace require a robotic system that can make maximum use of the limited workspace, and have enough flexibility to engage in most tasks, including large weight bearing activities. The glove box atmosphere is very dry and potentially dusty. A dry argon atmosphere creates problems in most motors because of electrical breakdown and motor brush arcing. 1 One solution is to have motor brushes treated for argon environment.

Dust causes several problems. Dust is abrasive to ball bearings, and sliding surfaces resulting in reduced reliability and limited lifetime. Solutions to these issues include sealing all motors and enclosures while keeping surfaces free of ridges and small grooves. Smooth surfaces help prevent dust accumulation. Radiation from dust particle poses a couple of problems. Concentrated accumulations of radioactive dust can be hazardous to operating personnel, so

\footnotetext{
1 Armantrout, Guy A. and Heywood, Ann C., "Robotic Requirements for Plutonium Handling Automation," IEEE Transactions on Nuclear Science, Vol. 37, No. 3, Junc 1990, pg. $1452-$ 1455.
} 
robot design must not encourage the accumulation of dust.

Radiation also is a concern for equipment reliability. Alpha radiation affects sensitive electronic components, and insulators, and causes general material degradation of all components. Such sensitive components must be isolated, hardened, or periodically replaced.

Other specific requirements for process automation include meeting extreme safety and security requirements, quality assurance and control standards. These specific requirements are due to the radioactive materials.

\section{Available Commercial Capabilities}

There are several alternatives available for acquiring a rohot with given special requirements for glove box automation. The three options evaluated are the purchase of a system built to specification, the in-house custom design using off the shelf components, or a cooperative development effort with the mariufacturer using existing products having proven reliability. The third option provides us with the most economical and mutually beneficial alternative. It benefits us as the customer because the manufacturer can provide the needed reliability data for the robotic system, while using their expertise in system development. In return the manufacturer learns what glove box processing needs exist and can enter a new market.

The system chosen was an overhead gantry robotic system because of the heavy workload requirements and the limited glove box workspace. This rail mounted rectilinear coordinate robotic arm can reliably participate in repetitive processes while making maximum use of the workspace, and allowing for future adaptions.

\section{Prototype Development}

This past year Lawrence Livermore National Laboratory has undertaken the intensive design and development effort of demonstrating the feasibility of an overhead gantry robotic system performing radioactive material processing. The robotic system manufacturer, IBM, has used their system development expertise coupled with LLNL's material processing expertise to develop multiple end of arm tools and processing workstations. These workstations were placeed into a simulated glove box. After extensive programming and tool placement the gantry robot successfully moved from workstation to workstation within the limited workspace, simulating typical material processes. This developmental test showed the feasibility of automating basic and repetitive material processes.

Upcoming developmental work includes placing this gantry mounted robotic system in a prototype glove box, simulating actual work loads, typical material processes, and realistic atmosphere conditions. Extensive nonnuclear material testing will be conducted to evaluate accessibility for maintenance and serviceability. Before this commercial robotic system is installed design upgrades will be implemented for environmental hardening. These upgrades include improved cabling systems, sealed motors and bearings, and radiation hardened electronic components.

There is another area of glove box automation that involves more complex and less repetitive operations. The need for more advanced machine tool control, operator interactive control systems, and the use of multiple manipulators is being investigated for situations where accuracy and safety are of higher concern than production. These situations require more sophisticated vision and control systems. In such situations a smaller articulating arm, or arms can be utilized. Initially these experiments will be 
conducted in an air environment to test operation concepts. The next step will be to test these special capabilities in a prototypical environment. Existing commercial robotic technology will be utilized to the fullest extent.

\section{Initial Observation}

When utilizing commercial robotic technology to satisfy the functional requirements of radioactive material processing in glove boxes, performance trade offs are evaluated. Such trade-offs include size versus weight lifting capacity, dust control versus serviceability of the unit, and system flexibility versus project objectives. The weight lifting capacity of a robot is directly proportional to the size of the robot. When placed into a glove box an articulating arm has limited range of motion and cannot lift the heavy workloads. The gantry system utilizes overhead space and can lift heavy loads in the vertical direction. To prevent excessive dust collection smooth surfaces and small release systems are preferred, but for ease of maintenance modular components and large release systems are needed. The system design must try to satisfy both of these requirements. Another trade-off is providing maximum system flexibility for future adaptations while keeping the present objectives in sight. This is most difficult because of unknown future demands. It is inevitable that some redesign must occur.

\section{Conclusions}

Automation of the DOE's nuclear complex is motivated by the increasing pressure to reduce operator dosage, minimize waste and guarantee security of nuclear materials.

Although commercial robotic systems are available having the required capabilities, they are not readily adaptable to a glove box environment. After careful evaluation of the available robotic systems, the overhead gantry robot was chosen because of its usage of maximum workspace, and its weight lifting capabilities. In conjunction with the manufacturer, Lawrence Livermore National Laboratory has successfully demonstrated the feasibility of robotics technology for radioactive material processing. The next phase of development will focus on safety, maintainability, and reliability. The next phase also includes the development and adaptation of teleoperation capabilities for situations requiring more complex operator interaction.

The adaptation of robotic systems to radioactive material processing introduce significant challenges, but those challenges motivate continuing design and development.

\section{Acknowledgements:}

Acknowledgements are made to all team participants who have made significant contributions to this development effort. Those participants are Lawrence Livermore National . Laboratory, IBM - Industrial Sector Division, Westinghouse Idaho Nuclear Corporation, Los Alamos National Laboratory, Sandia National Laboratory, and Scientific Applications International Corporation.

This work performed under the auspices of US DOE by LLNL under contract \#W-7405-eng-48.

\section{References:}

Armantrout, Guy A. and Heywood, Ann C., "Robotic Requirements for Plutonium Handling Automation," IEEE Transactions on Nuclear Science, Vol. 37, No. 3, June 1990, Pg. 1452 - 1455 

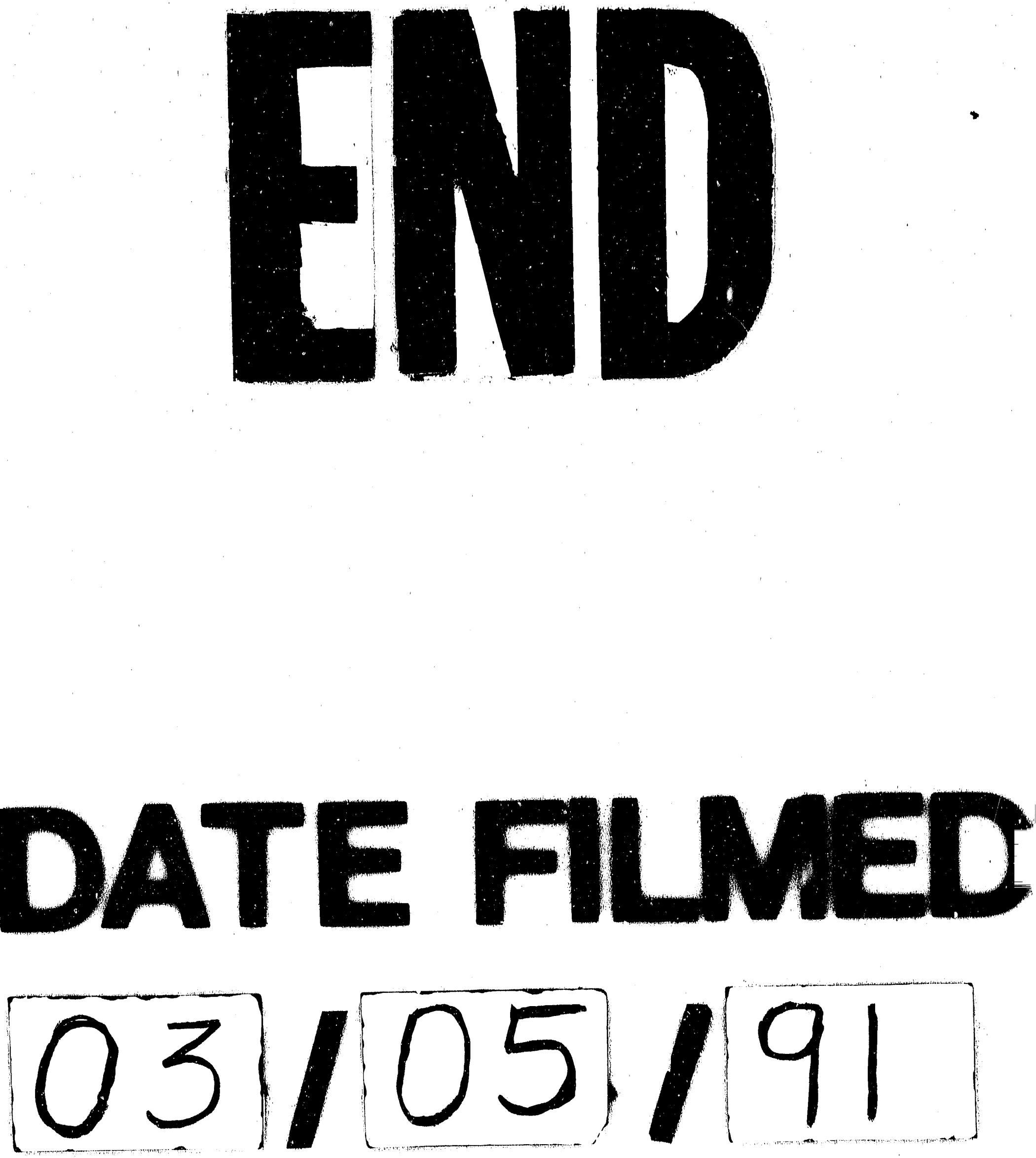
